\title{
Flow-Directed Catheters in Hepatic Embolization Therapy-A Review with Clinical Cases
}

\author{
Shams Iqbal ${ }^{1,2}$ Lawrence Jordan Breyfogle ${ }^{3} \quad$ Sebastian Flacke ${ }^{1}$ \\ ${ }^{1}$ Department of Radiology, Lahey Hospital \& Medical Center, \\ Burlington, Massachusetts, United States \\ 2Department of Radiology, Massachusetts General Hospital, Boston, \\ Massachusetts, United States \\ ${ }^{3}$ Department of Radiology, Tufts University School of Medicine, \\ Boston, Massachusetts, United States

\begin{abstract}
Address for correspondence Shams Iqbal, MD, FSIR, Department of Radiology, Massachusetts General Hospital, Boston, MA 02114, United States (e-mail: drshams241@gmail.com).
\end{abstract}

J Clin Interv Radiol ISVIR 2021;5:99-105.

\begin{abstract}
Keywords

- hepatic embolization

- flow-directed

catheters

- antireflux catheters

Transarterial embolization with chemotherapy and radiation is well-documented forms of treatment for liver cancers but reflux of embolic particles to nontarget tissues can result in unintended consequences such as gastrointestinal ulceration. Traditionally, operators have used coil embolization of hepatoenteric collaterals to prevent reflux. Antireflux microcatheters that contain expandable baskets (Surefire) or inflatable balloons have recently been developed as tools to avoid these side effects. We describe cases where antireflux catheters were used instead of coil embolization. Using antireflux catheters, we eliminated particle reflux into nontarget vessels. We also review the literature on antireflux catheters involved in preventing reflux during chemo- and radioembolization.
\end{abstract}

\section{Introduction}

Transarterial chemoembolization (TACE) and selective internal radiation therapy (SIRT) with ${ }^{90} \mathrm{Y}$ resin and glass microspheres are established treatments for unresectable liver cancers including primary hepatocellular carcinoma (HCC), liver-dominant metastasis, and intrahepatic cholangiocarcinoma. Serious complications during transarterial embolization of liver masses may occur as a result of unintended embolization to nontarget hepatic and extrahepatic tissues. One potential complication is gastrointestinal ulceration after nontarget embolization of hepatoenteric collaterals such as the gastroduodenal artery (GDA), right gastric artery, and right gastroepiploic artery, leading to damage of the gastric wall, duodenum, and pancreas. ${ }^{1,2}$ Nontarget embolization of a patent falciform artery may lead to burning sensations and pain in the umbilical and anterior abdominal wall region. ${ }^{3}$ Additionally, radiation and chemotherapy induced cholecystitis has also been reported from nontarget embolization after chemoembolization beads and ${ }^{90}$ Y treatment. ${ }^{4}$ One way this may occur is anterograde blood flow through hepatofugal arteries forming collaterals to nearby arteries and operators often have to navigate these variations in foregut vascular anatomy to prevent nontarget embolization. ${ }^{5}$

This already difficult anatomy may become even more complex after liver resection where small collateral vessel may develop and provide blood supply to small segments of the liver or drain blood from the remaining liver artery into neighboring organs such as the duodenum.

Also, refluxing embolic particles are a major source of nontarget embolization. Retrograde blood flow during diastole can carry particles to nearby arterial branch points,

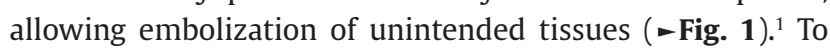
prevent reflux, interventionalists have conventionally used coil embolization in pretreatment planning before TACE and SIRT. Often, arteries involved in reflux are too small to place

(C) 2021. Indian Society of Vascular and Interventional Radiology. This is an open access article published by Thieme under the terms of the Creative Commons Attribution-NonDerivative-NonCommercial-License, permitting copying and reproduction so long as the original work is given appropriate credit. Contents may not be used for commercial purposes, or adapted, remixed, transformed or built upon. (https://creativecommons.org/licenses/by-nc-nd/4.0/). Thieme Medical and Scientific Publishers Pvt. Ltd. A-12, 2nd Floor, Sector 2, Noida-201301 UP, India published online

May 17, 2021
DOI https://doi.org/

$10.1055 / \mathrm{s}-0041-172949$ ISSN 2457-0214 


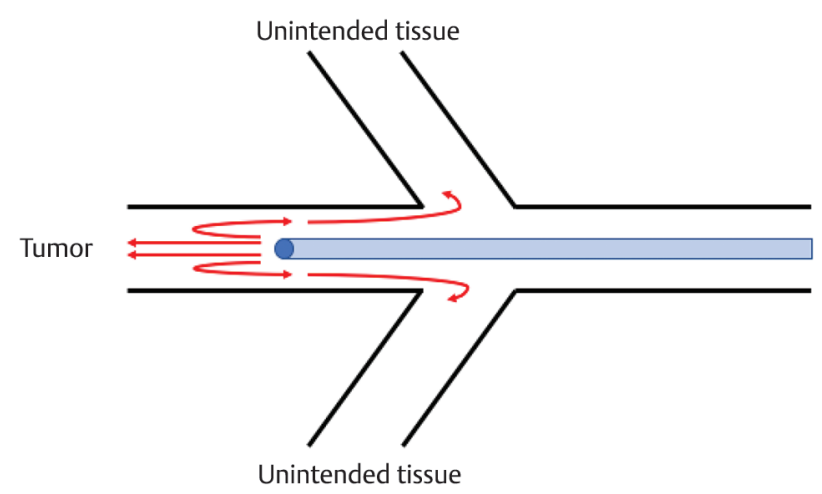

Fig. 1 Retrograde blood flow during diastole using a standard end-hole catheter to administer embolic particles. Blood flow is seen refluxing into nontarget tissues creating a potential source of complications.

coils into and on the day of treatment, the flow may have redistributed in a different way compared with the day of embolization. This calls for alternative ways to manage such situations, such as antireflux catheters. These catheters are designed to increase the relative deposition of embolic material to the target with temporary manipulation of the anatomy and physiology of the arterial system. At present, there are two kinds of antireflux catheters available, one with expandable basket catheter system and other with temporary occlusive balloon expandable micro catheters.

The Surefire Infusion System (Surefire Medical Inc. now TriSalus Life Sciences, Westminster, Colorado, United States) was developed as an antireflux alternative to the standard end-hole catheter and coil embolization for infusing embolic particles during TACE and SIRT. As seen in $\boldsymbol{- F i g . ~ 2 , ~ t h e ~ S u r e f i r e ~}$ catheter contains a flexible, cone-shaped self-expanding basket with a hydrophilic coating that collapses to allow anterograde flow during systolic flow. The antireflux basket will re-expand during diastolic retrograde blood flow that occludes the vessel and traps embolic particles thus preventing reflux. ${ }^{6,7}$

Temporary occlusion using balloon microcatheters has also been explored as a means to prevent particle reflux ( - Fig. 3). Balloon occlusion results in decreased vascular blood pressure in the tumor-supplying artery that reverses blood flow in adjacent hepatoenteric arteries. This mechanism has been demonstrated by Rose et al, which showed increased deposition of microspheres in target porcine hepatic arteries. ${ }^{8}$ There have been a few recent studies investigating the feasibility and efficacy of balloon catheter occlusion in preventing reflux, higher target bead packing and enhancing target embolization. Balloon devices currently being studied include IsoFlow (Vascular Designs Inc., San Jose, California, United States), Hyperform (Medtronic, Minneapolis, Minnesota, United States), Occlusafe microcatheter (Terumo, Japan), and Sniper (Embolx Inc., Sunnyvale, California, United States).

We present cases showing the clinical uses of antireflux catheters in patients undergoing hepatic artery chemoembolization and radio embolization. In addition, we review

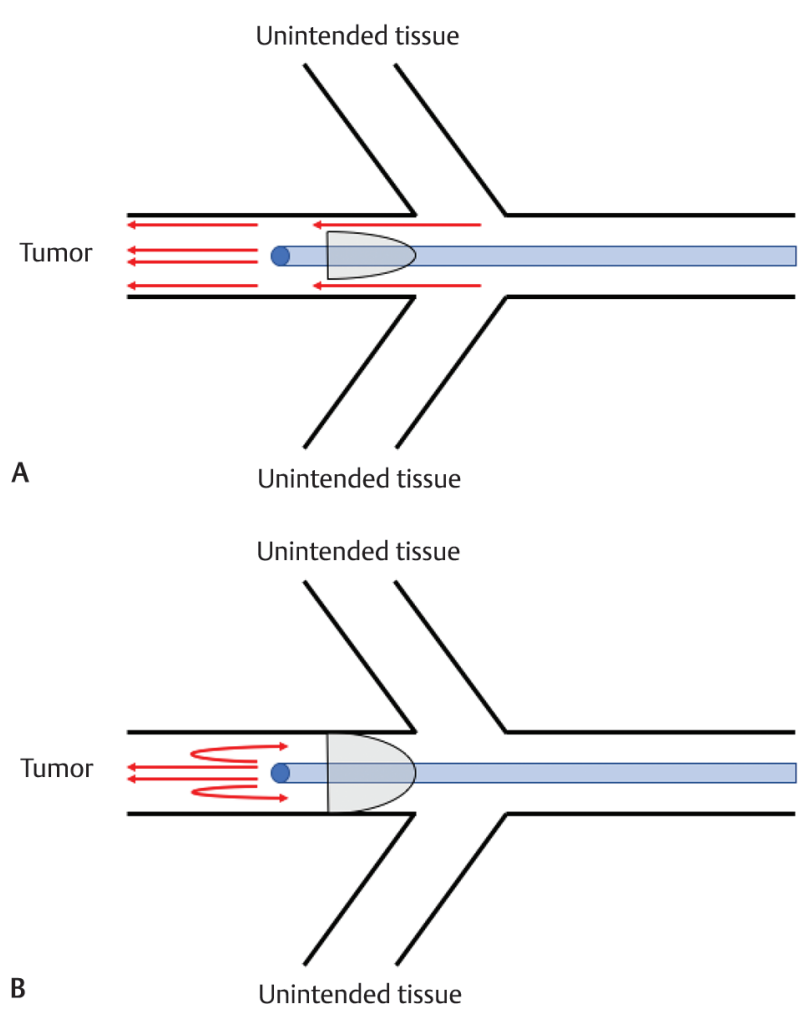

Fig. 2 Mechanism of Surefire antireflux microcatheter. (A) Systolic blood pressure collapses the antireflux basket to allow anterograde blood flow. (B) Diastolic blood pressure allows antireflux basket to expand and catch embolic particles, preventing reflux to unintended tissue.

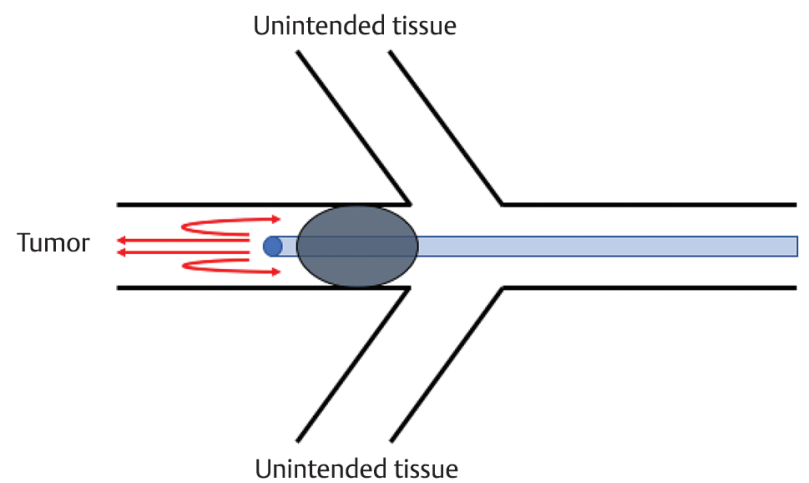

Fig. 3 Mechanism of balloon catheter occlusion. Inflation of balloon allows temporary arterial occlusion and prevents reflux to unintended tissues.

current literature on preventing reflux and enhancing downstream embolization using the Surefire microcatheter and balloon tip catheters.

\section{Case \#1: Surefire for Y 90 Therapy}

A 62-year-old woman presented with recurrent cholangiocarcinoma status post right hepatic lobectomy and chemoradiation therapy for her initial lesion. Surveillance imaging revealed a new solitary lesion in the fourth hepatic segment ( - Fig. 4). Biopsy was consistent with recurrent 


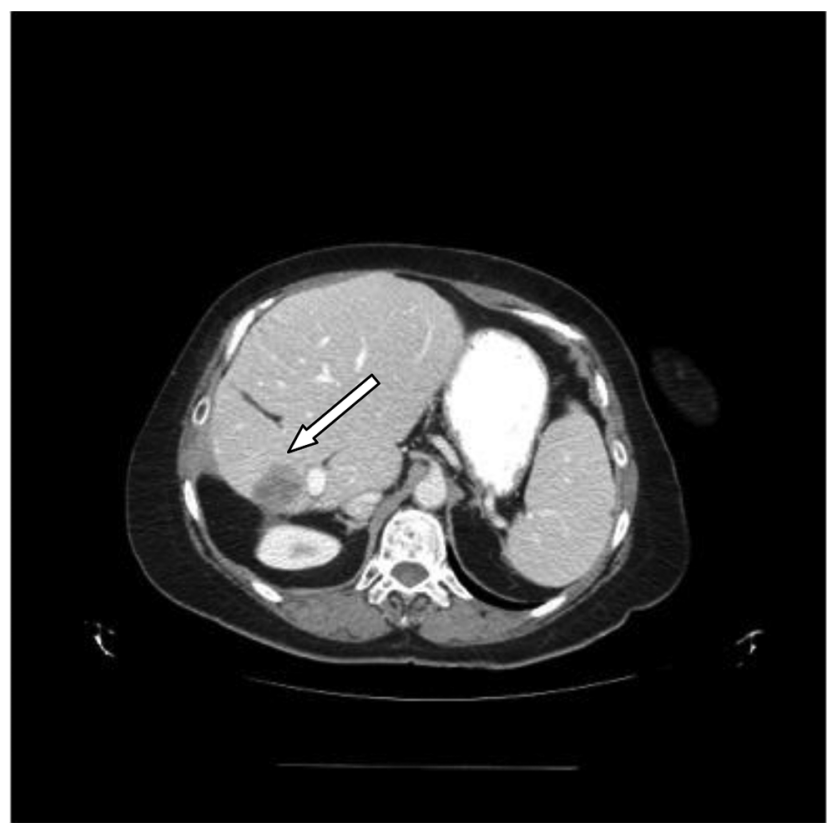

Fig. 4 A 62-year-old woman who presented with recurrent cholangiocarcinoma status post right hepatic lobectomy and chemo-radiation therapy for her initial lesion. Surveillance imaging revealed a new solitary lesion in the fourth hepatic segment (arrow). Patient is status post right hepatectomy.

cholangiocarcinoma. A multidisciplinary team decided to treat this tumor with SIRT given that with her previous surgery, she was unable to undergo further resection.

Pretreatment celiac angiogram revealed a replaced left hepatic artery that branches off the proximal celiac artery and provides the major blood supply to the hypertrophied left liver lobe ( - Fig. 5). The common hepatic artery branches off into GDA and two small parasitized collateral branches supplying segment 4 , which is the cut surface of the liver and bears the tumor. These two arteries showed competitive flow from the left hepatic artery and the GDA. On interrogation, the lateral of these branches supplies the tumor as shown by DynaCT; however, on forceful injection of contrast, there is reflux via intrahepatic collaterals into the medial segment 4 artery and retrograde flow into the GDA ( - Fig. $\mathbf{6}$ ). Attempts were made to engage these two small branches from the left hepatic artery; however, this was unsuccessful. This would leave the possibility of a nonselective injection of Y90 particles into the distal left hepatic artery with protective embolization of the two parasitized collateral branches or the GDA itself. However, the excellent opacification of the tumor after selective injection into the lateral collateral branch favored treatment through this branch with protection against unintended reflux. During treatment, a Surefire microcatheter was advanced into the lateral hypertrophied collateral branch vasospasm occurred after filter deployment that responded to $60 \mathrm{mg}$ of cardiac lidocaine. Repeated contrast injections showed arterial patency and no further vasospasm ( - Fig. 7). In contrast to pretreatment angiography, after filter deployment no reflux was seen. Subsequently, ${ }^{90} \mathrm{Y}$

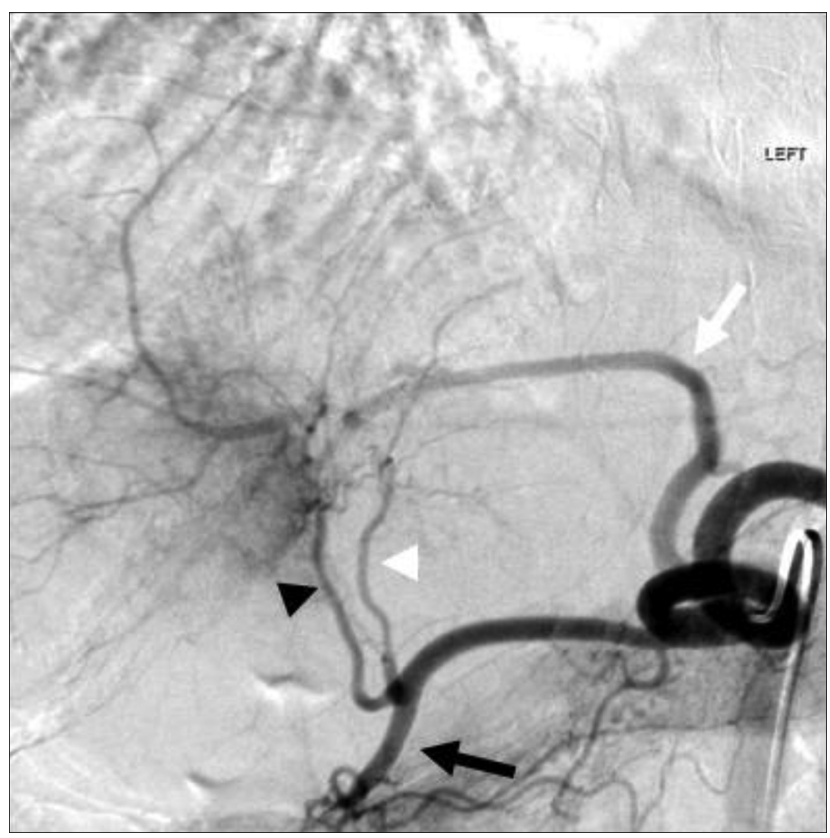

Fig. 5 A 62-year-old woman who presented with recurrent cholangiocarcinoma status post right hepatic lobectomy and chemoradiation therapy for her initial lesion. Surveillance imaging revealed a new solitary lesion in the fourth hepatic segment. Pretreatment angiography of the celiac trunk. Large white arrow represents replaced left hepatic artery. Large black arrow represents gastroduodenal artery. Black arrowhead represents lateral branching posterior hepatic artery supplying blood to tumor. White arrowhead represents medial branching posterior hepatic artery.

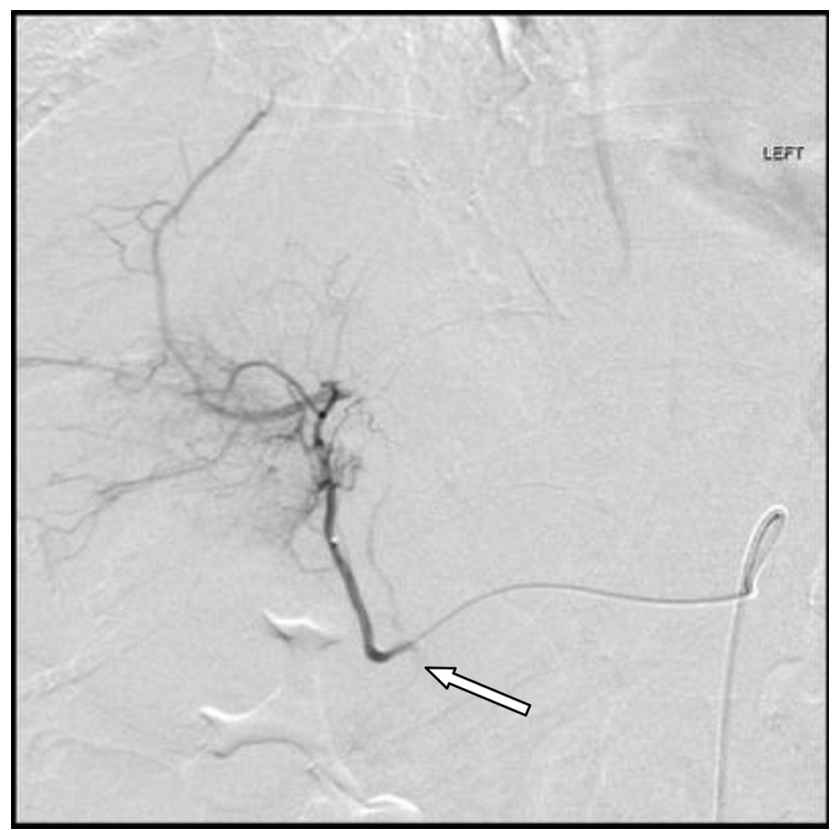

Fig. 6 A 62-year-old woman who presented with recurrent cholangiocarcinoma status post right hepatic lobectomy and chemoradiation therapy for her initial lesion. Surveillance imaging revealed a new solitary lesion in the fourth hepatic segment. Pretreatment angiography of the lateral branching posterior hepatic artery that supplies the tumor. Refluxing contrast is seen into gastroduodenal artery (arrow) via the medial branching middle hepatic artery. 


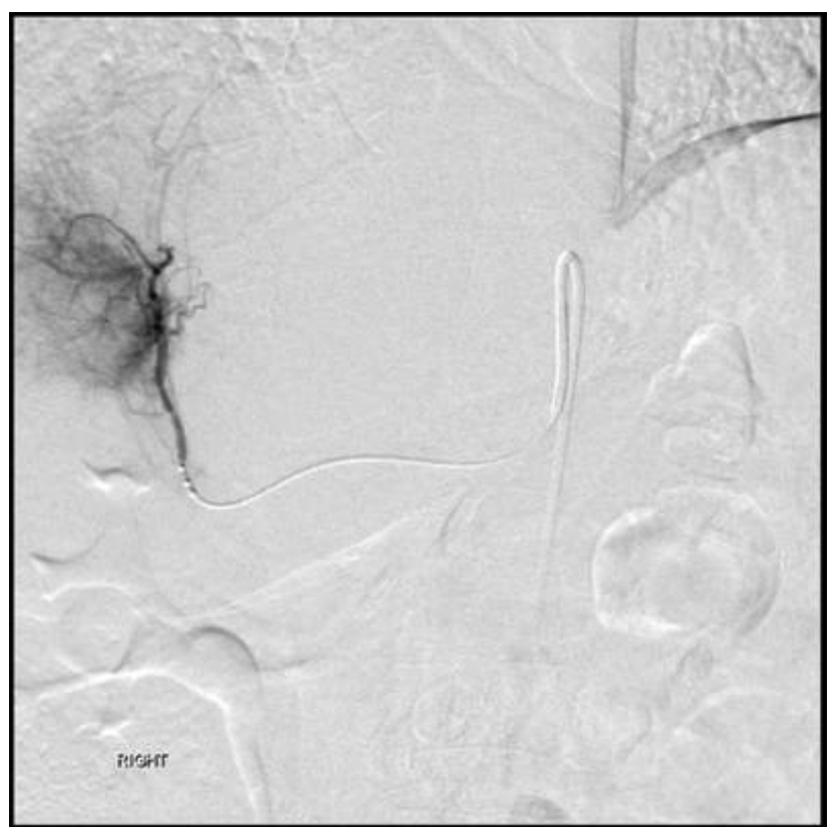

Fig. 7 A 62-year-old woman who presented with recurrent cholangiocarcinoma status post right hepatic lobectomy and chemoradiation therapy for her initial lesion. Surveillance imaging revealed a new solitary lesion in the fourth hepatic segment. Deployment of the Surefire antireflux catheter tip during ${ }^{90} \mathrm{Y}$ radioembolization. No evidence of reflux is seen in contrast to pretreatment angiography.

resin spheres were administered to the tumor through the Surefire microcatheter. Intermittent angiograms were obtained during ${ }^{90} \mathrm{Y}$ injection to assess persistent antegrade flow without reflux into the GDA to identify a suitable end-point of radioembolization.

Postembolization Bremsstrahlung scan did not show any extrahepatic activity. The 3-month follow-up scan showed extensive tumor necrosis. One year later, the patient developed intrahepatic metastatic disease and underwent a repeat SIRT. This time, as metastases were distributed throughout the entire left lobe; a nonselective treatment via the left hepatic artery was performed after embolization of the remaining medial collateral branch. The lateral collateral branch was completely occluded at the time of the repeat treatment.

\section{Case \# 2: Surefire for Chemoembolization}

A 59-year-old male presented with hepatitis C, treated briefly with Harvoni, underwent ultrasound that showed a mass. Follow-up magnetic resonance imaging (MRI) in February showed a $3.3 \mathrm{~cm}$ mass in the right lobe (-Fig. 8A), with delayed washout consistent with HCC. The patient was evaluated for liver transplant. As a bridge to liver transplant, chemoembolization of the liver was requested. Angiogram showed the blood supply to the lesion from right hepatic artery. To minimize the potential reflux of chemo beads, Surefire catheter was used ( $\mathbf{- F i g . ~ 8 B}$ ) that minimized staining of the surrounding area with intense staining of the tumor. Follow-up contrast-enhanced MRI showed no enhancement of the liver lesion with expected peripheral rim enhancement as posttreatment changes. The patient went on to undergo a successful orthotopic liver transplant.

\section{Case \# 3: Balloon Occlusion Catheter for Chemoembolization}

A 61-year-old male, with hepatitis B virus-related cirrhosis, presented with right upper quadrant pain and underwent cholecystectomy. Liver MRI was done showing liver lesions. MRI was inconclusive for diagnosis (-Fig. 9A). A biopsy was performed that proved it to be well differentiated HCC. Chemoembolization was requested. The lesion in segment 6 was targeted; however, with the end-hole catheter, there was significant reflux to the portal venous side via portal venous shunt. This was treated with Sniper balloon occlusion catheter (Embolx Inc., Sunnyvale, California, United States) with significant decrease in the portal venous shunting (-Fig. 9C). The patient is under follow-up and slated to undergo imaging next month.

\section{Technical Note}

The Surefire catheter and balloon embolization catheters are compatible with the present sheaths commercially available. We used 6 French sheaths. This allows flushing of the sheath using the side arm. The balloon occlusion catheters are compatible with routinely available 5 French catheters. Surefire catheter requires specific catheters that were provided by the
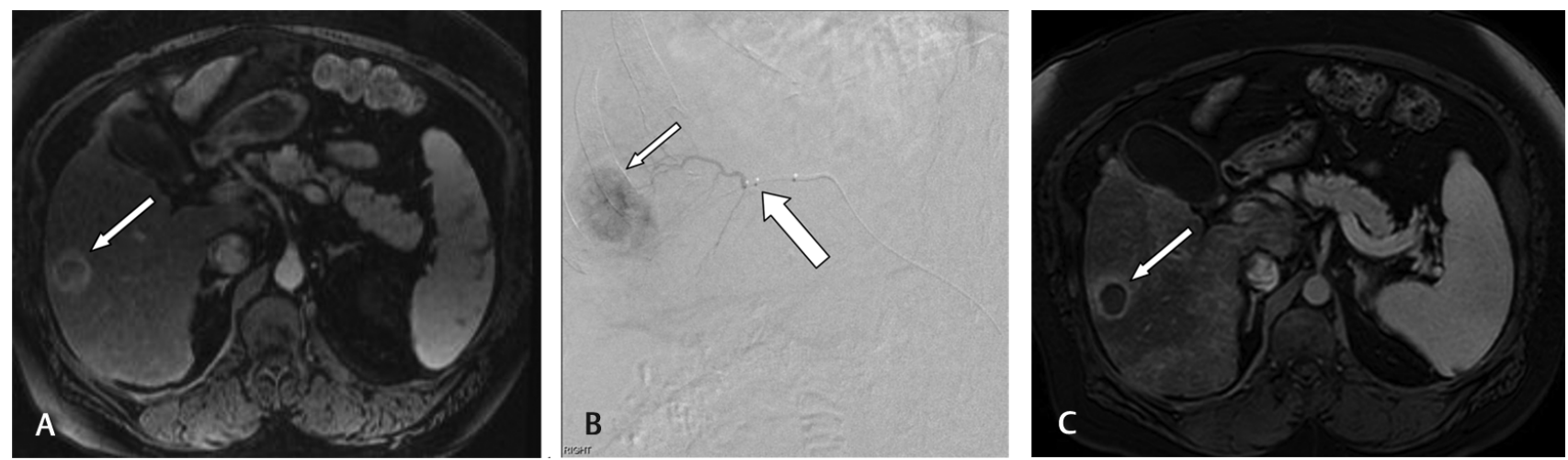

Fig. 8 Surefire catheter for chemoembolization. (A) Axial post contrast magnetic resonance imaging (MRI) of the liver showing an enhancing lesion in the right lobe (arrow). (B) Catheter angiogram using Surefire catheter (thick arrow) with intense staining of liver lesion. (C) Follow-up axial contrast-enhanced MRI showing no residual enhancement of the treated lesion (arrow). 

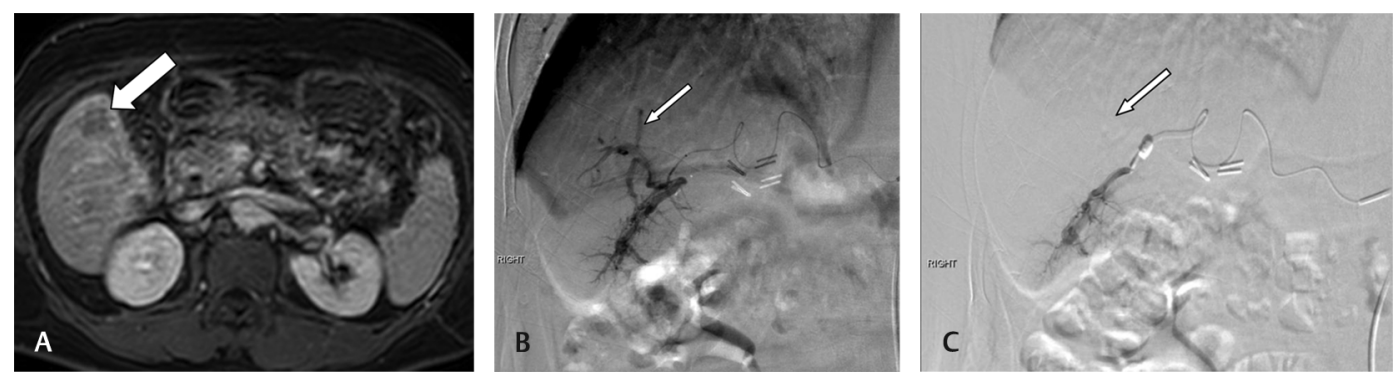

Fig. 9 A 61-year-old male with hepatitis B virus with incidentally found liver lesion and biopsy-proven hepatocellular carcinoma. (A) Axial post contrast magnetic resonance imaging showing the liver lesion in right lobe of liver (arrow). (B) Angiogram with regular end hole catheter showing significant shunting to the portal vein (thin arrow). (C) Angiogram with sniper balloon occlusion catheter showing significant decrease in the portal vein filling (thin arrow). The injection conditions of both end hole catheter and sniper were unchanged.

same company, in the usual shapes (Cobra, Simmons). These catheters have thinner walls, increasing the lumen size to accommodate the flow-directed catheters.

The Surefire catheter is prepped per the company guidelines, which is targeted toward avoiding any trapped air in the fabric of the device, thereby avoiding any distal air embolization.

\section{Discussion}

In this review, we describe a patient undergoing SIRT for recurrent cholangiocarcinoma with complex hepatoenteric anatomy. Pretreatment angiography showed reflux into GDA; thus, a decision was made to use antireflux catheter (Surefire) for the treatment.

Of the available antireflux catheters, Surefire has been studied the most, which is suggested by the number of publications involving it. The primary goals of antireflux catheters are to avoid reflux and increase the percentage distribution of embolized particles to the target versus nontarget tissues. However, whether this translates into decreased number of treatments or increased survival is yet to be proven.

The ability of the Surefire antireflux microcatheter to help embolization when there is complex hepatic anatomy has been previously documented; a case study by Saddekni et al highlights the success in delivering chemoembolization particles to downstream liver tumors despite a collateralizing variant retroportal artery with retrograde flow providing a problematic course for nontarget embolization. By using the antireflux catheter, the authors avoided obliteration of the flow through the collateral and successfully treated the patient with multifocal HCC. ${ }^{6}$

As demonstrated in our case study, the Surefire microcatheter can avoid the need to coil embolize as a means to prevent reflux and nontarget embolization. This is supported in a case study by van den Hoven et al where the authors were unable to coil embolize the right gastric artery yet successfully administered ${ }^{90} \mathrm{Y}$ using the Surefire system to prevent extrahepatic deposition. ${ }^{9}$ Fischman et al verified these findings in a prospective trial comparing nontarget embolization between coil embolization with an end-hole catheter and the Surefire antireflux catheter alone during pretreatment angiography in patients undergoing ${ }^{90} \mathrm{Y}$ radioembolization. In this prospective trial, 30 patients with primary or metastatic liver cancer underwent pretreatment angiography and SIRT. There was no nontarget distribution of radiotracer seen in either group after planning angiography and macroaggregated albumin (MAA) using single-photon emission computed tomography/computed tomography. The authors assert that the antireflux catheter is equally as good as coil embolization in reducing nontarget embolization with decreased procedure time, less radiation, and less contrast use while maintaining patency of collaterals by avoiding embolization. ${ }^{10}$ This was earlier proven in animal study, using a porcine renal artery model, by Arepally et al who showed statistically significantly decreases in nontarget deposition compared with the end-hole catheter using tantalum bead embolization. The authors propose that the nontargeted kidney pole received more bead with the end-hole catheter compared with the Surefire antireflux catheter as a result of more irregular particle distribution pattern with the end-hole catheter. ${ }^{11}$

Beyond preventing nontarget embolization, there is evidence that antireflux catheter enhances downstream particle delivery to liver tumors. Arepally et al describe deeper penetration of tantalum microspheres in downstream kidney parenchyma of porcine kidneys after infusion with an antireflux catheter compared with an end-hole catheter. The antireflux catheter had a statistically significantly increase in embolization efficiency (99.9\%) compared with end-hole catheter $(72 \%)$ in this in vivo model; and only $0.1 \%$ of tantalum microspheres exhibited arterial reflux with the antireflux catheter compared with $28 \%$ reflux to nontarget renal tissue with the end-hole catheter. ${ }^{11}$ These findings were further supported by the work of Pasciak et al, who treated unresectable liver cancer with same day MAA and Y90 treatments. They showed a significant decrease in nontarget deposition by 24 to $89 \%$ and statistically significant relative increase in tumor deposition by 33 to $90 \%{ }^{12}$ These findings were also shown to be consistent in drug-eluting bead transarterial chemoembolization (DEB-TACE) where larger particles are used for treatment, in comparison to the Y90 therapy. The retrospective study by Kim et al found no evidence of nontarget hepatotoxicity following DEB-TACE in all 22 patients using the Surefire Infusion System, suggesting that this antireflux catheter is a suitable and safe alternative to the end-hole catheter. ${ }^{13}$

The Surefire catheter has also been shown to change procedure parameters. According to Morshedi et al, using a Surefire antireflux catheter alone results in a statistically 
significant decrease in procedure time, fluoroscopy time, and contrast dose compared with coil embolization and end-hole catheter for nontarget embolization protection during pretreatment angiography before ${ }^{90} Y$. However, there was no significant decrease in radiation dose. A shorter procedure and fluoroscopy time increase efficiency and can decrease overall cost. However, there was no statistically significant decrease in radiation dose. In addition, a lower contrast dose could be beneficial for patients with renal disease. These findings may be beneficial to the patient in reducing risk and exposure to radiation, contrast material, and anesthesia. ${ }^{14}$

These findings are supported are by Fischman et al who found that the antireflux catheter alone can significantly decrease procedure time, fluoroscopy time, contrast agent dose, and radiation dose in pretreatment planning angiography for SIRT when compared with coil embolization with an end-hole catheter. ${ }^{10}$

\section{Mechanism of Action}

The mechanism of action of the antireflux catheter is multifactorial and more complex than simple expansion of basket to prevent particle reflux. Rose et al investigating alterations in blood pressure in hepatoenteric arterial flow discovered that when the antireflux tip expands during diastolic retrograde flow, it occludes the arterial lumen and creates two separate vascular compartments-a lower pressure compartment distal to the catheter tip and a higher pressured systemic arterial compartment. There are significant decreases in systolic, diastolic, and mean arterial blood pressure in the hepatic arterial system when the antireflux tip is expanded versus closed (mean SBP decreased by 25; mean DBP decreased by 17; MAP decreased by 21 ). It has been suggested that the Surefire catheter is most efficacious in decreasing distal pressure in arteries with an internal diameter less than $4 \mathrm{~mm} .^{7}$ The authors suggest that there is a decrease in downstream hepatic arterial blood pressure when the antireflux catheter tip is expanded potentially causes hepatopedal flow in hepatoenteric arteries, reducing nontarget embolization. This provides retrograde protection through reflux prevention and anterograde protection through reversal of hepatoenteric flow. ${ }^{7}$ In theory, as the expandable tip collapses during systolic flow, the increased pressure difference may enhance embolic particle distribution into more distal target arteries.

This was further supported by an in-vitro hepatic arterial model, by van den Hoven et al showing differences in particle flow physics between end-hole catheters and the Surefire antireflux catheter. The authors assert that because the end-hole catheter results in more laminar particle outflow, and that normal arterial blood flow is laminar, downstream particle distribution with an end-hole catheter is more reliant upon catheter position within the arterial lumen. Therefore, distribution of embolization particles is more heterogeneous, and downstream spread may be subjected error and "streamlined" by pre-existing pathways of blood flow. Infusion with the antireflux catheter results in turbulent flow that may cause lateralization of embolic particles and lead to a more homogenous downstream particle distribution. The ability of the Surefire basket to disrupt laminar flow may lead to improved target radioembolization. ${ }^{15}$

\section{Limitations}

Use of antireflux catheters has some limitations in transarterial embolization. Initially, for the Surefire antireflux vessel diameter and access guiding catheters were limitation since it needed a 6 French guiding catheter; however, with the advent of newer microcatheter systems and Surefire-compatible guiding catheters, these have been circumvented. In a study by Rose et al, $33 \%$ of the initial study candidates were excluded from participation as the guide sheath could not fit due to aortoceliac or aorto-superior mesenteric artery anatomy. ${ }^{7}$ This may be related to the stiffness of the guide sheath. ${ }^{14}$

The use of the Surefire antireflux catheter also changes the definition of embolization end-point. ${ }^{6}$ Conventionally, operators use fluoroscopic reflux of contrast material during embolization as an indication to stop infusion. Because the antireflux catheter prevents reflux, it may be difficult to identify an end-point and this could lead to over embolization. ${ }^{7}$ Kim et al have developed a method of optimizing embolization end points: routinely retract the tip and perform angiograms to assess anterograde flow at intervals of every one-half vial of infusion particles after two vials and stop infusion when contrast reflux is seen despite tip expansion. ${ }^{13}$ We used this same method in our case study that was satisfactory. This approach is operator-dependent, so there is a need for quantifying and standardizing the embolization end-points, especially to eliminate variation and bias when performing systematic reviews.

Multiple studies have reported that the Surefire antireflux catheter is associated with distal hepatic arterial vasospasm that has led to the use of nitroglycerin and verapamil infusions during embolization. ${ }^{9,10}$ In our study, a vasospasm was seen after deployment of the Surefire tip in which we used cardiac lidocaine to abate. It is unclear what effect this may have on embolization procedures and particle distribution, if any.

A significant issue is that, while there is clear evidence that the Surefire system enhances downstream embolization and inhibits reflux, there is no data suggesting survival benefit. While this catheter helped us treat the patient with difficult hepatic arterial anatomy, her cancer unfortunately returned in the same location.

Further limitations to the literature presented here include problems with heterogeneous patient populations. Many of the studies are small case studies, or retrospective studies with variable demographics, making assessment difficult. In addition, it seems that the antireflux catheter has a higher learning curve that may make standardization of TACE and transarterial radioembolization studies and treatments harder to accomplish at first, if using the Surefire catheter becomes more routine. ${ }^{10}$

There is concern that the antireflux catheters are more expensive compared with the traditional end-hole catheter, coil embolization, and alternative techniques. However, analysis from Morshedi et al suggests that the Surefire antireflux 
catheter leads to a lower cost compared with procedures requiring coil embolization due to decreases in procedure and fluoroscopy time. ${ }^{14}$ This is difficult to quantify as the cost and supply of different equipment vary from institution to institution and region to region around the world.

Recent research has determined that temporary occlusion using balloon microcatheters is a safe and feasible means of preventing reflux and increasing downstream particle distribution in TACE and SIRT. ${ }^{8,16-18}$ While there is no data suggesting balloon catheters increase survival benefit in embolization therapy, they have been shown to successfully navigate complex anatomy and prevent reflux. For example, Hagspiel et al could temporarily occlude the cystic artery during ${ }^{90} \mathrm{Y}$ treatment. ${ }^{18}$ While balloon microcatheters are expensive, they are significantly less costly than the Surefire microcatheter. To summarize the limitations, the flow-directed catheters are difficult to handle in tortuous anatomy, increase risk of spasm and dissection especially in patients receiving antiangiogenetic therapies, and with long inflation time can lead to parent vessel thrombosis. ${ }^{19}$

In summary, using the antireflux catheter is useful in avoiding reflux in patients during liver-directed arterial therapies. We successfully treated patients with complex hepatoenteric anatomy and minimized unintended reflux. While the antireflux systems have been shown to prevent reflux, increase target embolization, and downstage certain HCC patients for potential liver transplant, it has limitations that mainly include cost and lack of a proven survival benefit. Temporary occlusion using balloon microcatheters may prove to be a less expensive and effective means to avoid reflux and increase downstream particle uptake in liver tumors. More research is needed to look at survival benefit in patients with using nontraditional, antireflux devices, and compare them for efficacy and ultimately survival benefit.

\section{Conflict of Interest}

None declared.

\section{References}

1 Mallach S, Ramp U, Erhardt A, Schmitt M, Häussinger D. An uncommon cause of gastro-duodenal ulceration. World J Gastroenterol 2008;14(16):2593-2595

2 Ingraham CR, Johnson GE, Nair AV, Padia SA. Nontarget embolization complicating transarterial chemoembolization in a patient with hepatocellular carcinoma. Semin Intervent Radiol 2011;28(2):202-206

3 Bhalani SM, Lewandowski RJ. Radioembolization complicated by nontarget embolization to the falciform artery. Semin Intervent Radiol 2011;28(2):234-239

4 Hickey R, Lewandowski RJ. Hepatic radioembolization complicated by radiation cholecystitis. Semin Intervent Radiol 2011;28(2):230-233
5 Murthy R, Brown DB, Salem R, et al. Gastrointestinal complications associated with hepatic arterial Yttrium-90 microsphere therapy. J Vasc Interv Radiol 2007;18(4):553-561, quiz 562

6 Saddekni S, Moustafa AS, Moawad S, Mahmoud K, Hamed BF, Abdel-Aal AK. Approaches for safe transarterial chemoembolization of multifocal hepatocellular carcinoma with retrograde flow in a retroportal artery. Radiol Case Rep 2017;13(1):171-174

7 Rose SC, Kikolski SG, Chomas JE. Downstream hepatic arterial blood pressure changes caused by deployment of the surefire antireflux expandable tip. Cardiovasc Intervent Radiol 2013;36(5):1262-1269

8 Rose SC, Halstead GD, Narsinh KH. Pressure-directed embolization of hepatic arteries in a Porcine model using a temporary occlusion balloon microcatheter: proof of concept. Cardiovasc Intervent Radiol 2017;40(11):1769-1776

9 van den Hoven AF, Prince JF, Samim M, et al. Posttreatment PET-CT-confirmed intrahepatic radioembolization performed without coil embolization, by using the antireflux Surefire infusion system. Cardiovasc Intervent Radiol 2014;37(2):523-528

10 Fischman AM, Ward TJ, Patel RS, et al. Prospective, randomized study of coil embolization versus Surefire infusion system during yttrium-90 radioembolization with resin microspheres. J Vasc Interv Radiol 2014;25(11):1709-1716

11 Arepally A, Chomas J, Kraitchman D, Hong K. Quantification and reduction of reflux during embolotherapy using an antireflux catheter and tantalum microspheres: ex vivo analysis. J Vasc Interv Radiol 2013;24(4):575-580

12 Pasciak AS, McElmurray JH, Bourgeois AC, Heidel RE, Bradley YC. The impact of an antireflux catheter on target volume particulate distribution in liver-directed embolotherapy: a pilot study. J Vasc Interv Radiol 2015;26(5):660-669

13 Kim AY, Frantz S, Krishnan P, et al. Short-term imaging response after drug-eluting embolic trans-arterial chemoembolization delivered with the Surefire Infusion System ${ }^{\circledR}$ for the treatment of hepatocellular carcinoma. PLoS One 2017;12(9):e0183861

14 Morshedi MM, Bauman M, RoseSC, KikolskiSG. Yttrium-90 resin microsphere radioembolization using an antireflux catheter: an alternative to traditional coil embolization for nontarget protection. Cardiovasc Intervent Radiol 2015;38(2):381-388

15 van den Hoven AF, Lam MG, Jernigan S, van den Bosch MA, Buckner GD. Innovation in catheter design for intra-arterial liver cancer treatments results in favorable particle-fluid dynamics. J Exp Clin Cancer Res 2015;34:74

16 Monsky WL, Padia SA, Hardy AH. Dual-balloon infusion microcatheter for selective drug-eluting bead transarterial chemoembolization: initial feasibility study. Diagn Interv Radiol 2017;23(6):454-460

17 Saltarelli A, Pelle G, Notarinanni E, Pasqualini V, Cianni R, Goretti SM. Y90 radioembolization with Occlusafe catheter infusion system in patients with unresectable hepatic metastasis. J Vasc Interv Radiol 2017;28:S213-S214

18 Hagspiel KD, Nambiar A, Hagspiel LM, Ahmad EA, Bozlar U. Temporary arterial balloon occlusion as an adjunct to Yttrium-90 radioembolization. Cardiovasc Intervent Radiol 2013;36(3):809-813

19 Kim HC. Balloon-occluded transarterial chemoembolization: hot air or hot stuff? J Vasc Interv Radiol 2019;30(3):347-348 